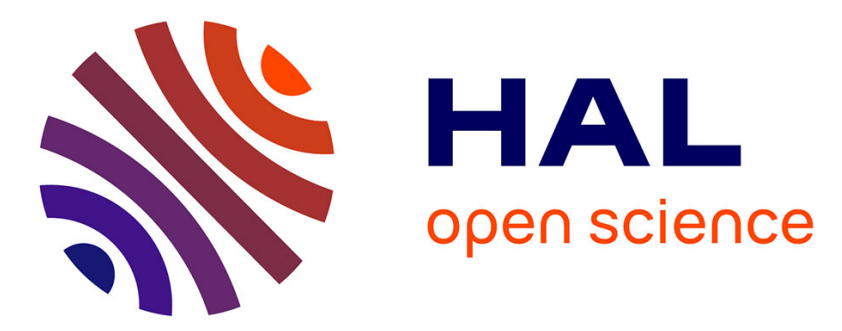

\title{
A la source du futur: premières formes verbales dans les productions spontanées de deux enfants français de 18 mois à 3 ans
}

Aliyah Morgenstern, Christophe Parisse, Martine Sekali

\section{- To cite this version:}

Aliyah Morgenstern, Christophe Parisse, Martine Sekali. A la source du futur: premières formes verbales dans les productions spontanées de deux enfants français de 18 mois à 3 ans. Faits de langues, 2010, 33, pp.163-176. 10.1163/19589514-033-01-900000019 . halshs-00495644

\section{HAL Id: halshs-00495644 \\ https://shs.hal.science/halshs-00495644}

Submitted on 28 Jun 2010

HAL is a multi-disciplinary open access archive for the deposit and dissemination of scientific research documents, whether they are published or not. The documents may come from teaching and research institutions in France or abroad, or from public or private research centers.
L'archive ouverte pluridisciplinaire HAL, est destinée au dépôt et à la diffusion de documents scientifiques de niveau recherche, publiés ou non, émanant des établissements d'enseignement et de recherche français ou étrangers, des laboratoires publics ou privés. 


\title{
A la source du futur Premières formes verbales dans les productions spontanées de deux enfants français de 18 mois à 3 ans
}

\author{
Aliyah Morgenstern, Christophe Parisse et Martine Sekali
}

Le futur semble avoir un statut particulier dans de nombreuses langues naturelles, différent des autres temps grammaticaux, parfois absent de la conjugaison (en anglais par exemple), au point que l'on se demande s'il s'agit vraiment d'un temps grammatical (Lyons, 1978 ; Palmer 1979). L'étude des marques du futur dans le langage des enfants, ce que leur appropriation progressive révèle des rapports qu'ils entretiennent avec la notion de temps (objectif ou subjectif) peut nous apporter un éclairage intéressant. Par ailleurs, de nombreux observateurs constatent une baisse de la fréquence des emplois du futur simple en faveur du futur périphrastique en français et parfois déplorent ce qu'ils considèrent comme la perte d'une certaine richesse langagière. On peut aussi penser que l'on retrouve de manière cyclique un phénomène qui s'était déjà produit dans l'histoire de la langue puisque le futur simple français est issu du futur périphrastique latin ${ }^{1}$.

Comme le rappelle François (1984), la grammaire des locuteurs, et en particulier celle des enfants, ne coïncide pas forcément avec celle des linguistes. Mais nous pouvons essayer d'aller à la rencontre de la grammaire en constante évolution de l'enfant pour définir les marqueurs de futur par rapport à leur fonction en discours, au contexte, aux genres discursifs dans lesquels ils sont employés, à leur fréquence et leur distribution. L'analyse de données enfantines peut également nous donner des indications sur la présence et la concurrence des formes de futur marquées sur le groupe verbal en français.

L'enfant et la notion de temps

Les marqueurs temporels peuvent être considérés comme la trace de la conscience qu'a l'énonciateur de la relation entre la situation d'énonciation et la

${ }^{1}$ En dehors de la première et de la deuxième personne du pluriel qui ont été construites par analogie avec le présent. 
situation dont il parle. Dans le développement de l'enfant, c'est essentiellement le langage qui permet de marquer et d'organiser l'extension des perspectives temporelles en même temps qu'il en témoigne.

On dit de l'enfant que son langage est d'abord ancré dans la deixis. De nombreux travaux décrivent et analysent l'expression du passé en tant que capacité à s'extraire du moment présent. Les compétences narratives se mettraient en place chez l'enfant entre les âges de trois et cinq ans (Applebee, 1978), mais les premiers récits apparaissent plus tôt et permettent à l'enfant de donner un sens à ses expériences. Vers l'âge de deux ans, les enfants commencent déjà à parler non seulement de ce qui les entoure, des actions en cours, mais à se déplacer mentalement dans le temps et dans l'espace et à raconter des évènements survenus ailleurs et dans le passé. Or tout comme le passé, le futur est du " non-présent », mais peut paraître plus complexe à maîtriser puisqu'il s'agit d'exprimer des évènements et des états sans en en avoir eu l'expérience, le vécu, et de les inscrire dans un temps postérieur au moment d'énonciation. C'est à ce titre que le futur est décrit (Lyons 1977², Comrie 1985³) comme étant au croisement du temps, de l'aspect et de la modalité et implique un positionnement énonciatif pour exprimer le degré d'éventualité que le procès advienne (modalité) ainsi que les liens de continuité, de différenciation ou de rupture par rapport au moment présent (aspect).

Pour parler du passé, il faut que l'enfant puisse se reposer sur sa mémoire et stabiliser ses représentations. Qu'en est-il du futur ? On sait que pour Piaget (1916, 1937), le schème pratique du temps s'élabore dès l'intelligence sensorimotrice, dans « la coordination des mouvements (...) dont l'ordre temporel se confond avec celui des déplacements » (1916, p. 270). Suite à ces travaux, l'école de Genève analysera essentiellement la construction de l'ordre temporel, des notions de successivité et de durée dans leur rapport avec le développement cognitif, ce qui permet à Piaget d'affirmer dans une perspective objectiviste que « comprendre le temps, c'est s'affranchir du présent» (op. cit. p. 274). Mais à cette analyse du rapport de l'enfant avec le temps, on peut superposer les travaux de Wallon (1945) et Malrieu (1953) pour qui objectif et subjectif sont interdépendants dans la construction de l'horizon temporel de l'enfant. Pour Wallon, l'origine du temps est affective et liée à l'insatisfaction : « (ce) sont des impatiences, qui sont fonction d'un besoin, d'une souffrance, d'un désir, d'une

2 « Le futur n’est jamais un concept purement temporel. Il inclut forcément un élément de prédiction ou une notion modale apparentée » (Lyons, 1987, traduction 1980, p. 299).

3 « The future is necessarily speculative in that any prediction we make about the future might be changed by intervening events, including our own conscious intervention. » (Comrie, 1985, p. 43.) 
attente, c'est-à-dire d'une orientation vers des objets et des évènements » (1945, p. 6). Pour Malrieu, les conduites à l'origine de la conscience du temps sont éminemment sociales. Le désir permet à l'enfant d'échapper à « l'éparpillement primitif par la tension vers le futur proche » (op.cit., p. 15). Il semble d'ailleurs y avoir un rapport très fort entre le désir et l'expression du futur dans un grand nombre de langues indo-européennes et non indo-européennes, « le futur est issu de formes qui à l'origine exprimaient le désir ou l'intention » (Lyons 1978, traduction 1980). Le futur serait donc une forme grammaticalisée de l'expression du désir et de l'intention.

\section{Les formes du futur dans le langage de l'enfant}

Dans leur forte dépendance à l'égard de la socialisation et du rôle des adultes, dans la mise en place de routines et d'habitudes qui sont aussi là pour provoquer des occasions de les rompre, continuités et ruptures permettant de façonner la notion de temps, passé et futur sont fortement liés. Il est donc particulièrement surprenant que l'expression du futur ait été relativement peu étudiée dans les travaux sur l'acquisition du langage chez l'enfant. On sait cependant qu'en français, les premières références au futur apparaissent de manière progressive dans le langage de l'enfant et sous des formes variées : d'une part des adverbes de temps, des prépositions, des subordonnées introduites par des conjonctions, et d'autre part le futur périphrastique et le futur simple.

Selon les travaux de Sabeau-Jouannet (1973), avant 2;06, les utilisations du futur périphrastique sont aspectuelles. (Exemple : « moi va ouvrir ton sac » sans se lever, l'enfant tend la main vers le sac, le prend et l'ouvre. Il s'agit de l'expression d'un projet ancré dans la situation, avec un début de réalisation de la part de l'enfant agent du procès). Les premières formes de temporalité future explicitement marquée se font vers 2;06 avec des adverbes mais aussi des formes de futur périphrastique allié à une préposition (" après moi va les enlever " l'enfant parle de chaussures qu'il n'a pas encore mises) où l'on peut parler de structuration temporelle. Le futur simple sera utilisé peu de temps après. L'appropriation de la référence au futur se ferait donc en quatre temps selon les données de Sabeau-Jouannet :

1) Autour de deux ans, l'enfant adhèrerait à la situation d'énonciation et ferait simplement l'opposition entre accompli et duratif (é tombé / tombe).

2) Vers 2;06, l'enfant marquerait l'opposition entre l'actuel et l'inactuel. Il y aurait une indifférenciation entre ce qui est de l'ordre du maintenant et de l'ordre du pas maintenant. C’est déjà ce que remarquait Grégoire (1947) chez son fils 
Charles qui dans le milieu de sa troisième année pouvait produire : « il fera dodo bébé hier » / « demain, nous on a vu les voitures ».

3) Vers 3 ans, l'enfant fait clairement l'opposition entre antériorité et postériorité mais seulement par rapport au moment d'énonciation. A cet âge sont attestées 27 formes de futur simple sur 67 formes de futur alors qu'à 2;02 elles étaient inexistantes sur 14 formes de futur.

4) L'enfant est capable vers $4 / 5$ ans de parler d'antériorité et de postériorité repérées par rapport à un temps en rupture avec le moment d'énonciation.

L'analyse de Sabeau-Jouannet va dans le même sens que les observations sur le français oral spontané adulte de JeanJean (1988) pour qui le futur périphrastique est très proche du présent simple (la substitution est souvent possible) puisqu'il s'agit d'une "visée prospective située dans le présent » (p. 241) qui " situe le procès dans le réel ". Ainsi, pour cette auteure, «morphologiquement et syntaxiquement ce futur est un présent ». D’autre part, le futur simple qui « situe le procès dans l'avenir » avec ancrage dans le virtuel, s'accompagnerait presque toujours, soit de compléments de temps, soit d'éléments appréciatifs, argumentatifs ou métalinguistiques.

Par ailleurs, on trouve des études sur la langue anglaise (Herriot 1969, Harner 1980) qui montrent malgré des résultats contrastés, que la compréhension du futur est problématique à l'âge de trois ans. La capacité à opérer un décentrage temporel s'améliore progressivement jusqu'à l'âge de 7 ans. Ces auteurs considèrent que les adverbes « before » et " after » sont compris plus tôt que les marqueurs de morphologie verbale. Par contre, la compréhension du futur en « will » et « going to » ne serait pas différenciée. Selon Gee (1985) cependant, il y aurait une distinction très nette entre les emplois de « will » et « gonna » chez les enfants américains dont elle a analysé les productions. Avec "gonna " l'enfant fait des projets qui ne seront pas forcément réalisés et qui sont le plus souvent en rupture avec la situation d'énonciation, alors qu'avec "will», l'enfant en collaboration avec l'adulte met en place les réalisations à venir de façon étroitement liée au contexte d'énonciation.

Cependant, comme le montrent les études d'Agnès Celle (1994, 1997, 2003) par exemple, on ne peut pas comparer systématiquement le futur simple et le futur périphrastique du français aux emplois de " will » et " going to » en anglais. Nos premières observations de données d'enfants anglophones confirment que l'appropriation des marqueurs n'est pas la même en anglais et en français, et que ces derniers sont chargés de valeurs différentes et souvent contraires à l'intuition du traducteur puisque le futur périphrastique, qui semble traduire «gonna» réfère plutôt à des actions en lien avec le moment 
d'énonciation, et le futur simple, que l'on traduit souvent avec le modal « will » à des évènements en rupture avec le moment d'énonciation. Le contraste entre le futur périphrastique et le futur simple en français, que cela soit au niveau de la fréquence d'utilisation, de l'ordre d'apparition, et des contextes dans lesquels ils sont utilisés est assez important.

Aussi, afin de discuter les propositions et positions de ces travaux, nous analyserons les premières références à l'avenir dans deux corpus longitudinaux d'enfants français, Léonard et Madeleine, filmés une fois par mois entre l'âge de 1;06 et 3 ans. Toutes les transcriptions ont été faites avec le logiciel CLAN en format CHAT avec alignement à la vidéo et possibilité d'utiliser les fonctions de $\mathrm{CLAN}^{4}$ pour faire les analyses quantitatives. Nous proposerons des jalons et des hypothèses sur l'expression du futur chez l'enfant en repérant l'émergence des formes et en faisant l'analyse de leur distribution, de leur fréquence et de leurs emplois en contexte.

Rappelons ce qu'écrit Paul Guillaume (1924) : « Ce n’est pas la présence de telle ou telle forme munie d'une marque grammaticale qui est importante mais son fonctionnement à l'intérieur du langage d'un enfant ». Cette observation est à mettre en lien avec les précautions que rappelle Franckel (1984, p. 65) : « On confond le futur en tant que marque et l'expression de l'avenir qui ne peut être construite que par l'énoncé tout entier, considéré dans ses conditions d'énonciation pertinentes ». Dans le cas de l'enfant, on insistera donc sur l'importance de l'inscription dialogique et co-énonciative de ses énoncés « tout entiers » et de leur articulation à ceux de leurs interlocuteurs adultes.

\section{FORMES}

Nous nous sommes concentrés sur l'expression du futur au niveau verbal. En effet, nous remarquons que chez les deux enfants, le futur périphrastique apparaît très tôt, à 2;01. Les adverbes de temps (" tout à l'heure ") ou les prépositions (" après ") apparaissent quasi simultanément mais de façon très sporadique au début et se combinent très rapidement aux formes verbales. Contrairement à ce que l'on pourrait penser, le futur simple est " vivant » et apparaît avant l'âge de trois ans chez ces deux enfants français du vingt-etunième siècle. Il nous faut ajouter que le développement linguistique de ces deux enfants est un peu au-dessus de la moyenne et qu'ils vivent dans un milieu social privilégié.

${ }^{4}$ Mac Whinney 2000 et http://childes.psy.cmu.edu/ 
Madeleine, qui a un langage très riche et varié dès le commencement du corpus emploie le futur simple dès 2;02 mais dans un contexte de sollicitation, et ses productions spontanées et plus fréquentes du futur simple se font ensuite à partir de 2;07. Léonard emploie le futur simple à partir de 2;11.

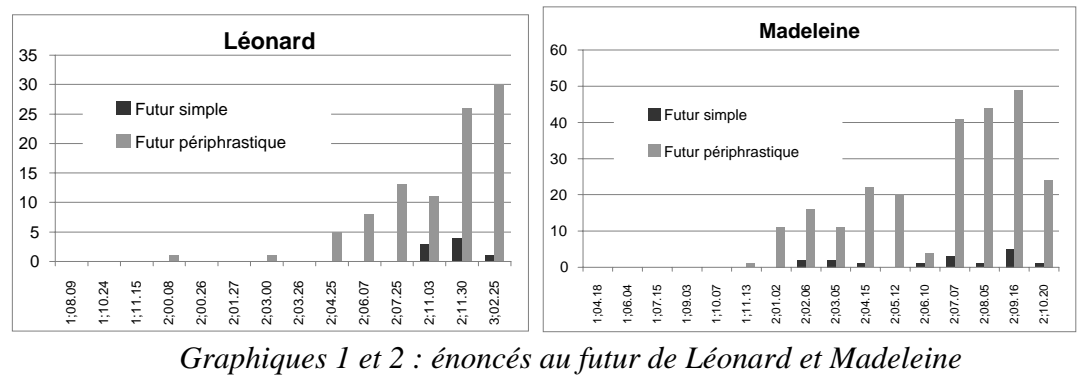

Au niveau quantitatif, il est intéressant de constater que les enfants utilisent sur toute la période concernée quatorze (Madeleine) et quinze (Léonard) fois plus de futur périphrastique que de futur simple. Nous avons voulu savoir si ce résultat était lié au langage entendu. Effectivement, les adultes emploient quant à eux cinq (interlocuteurs de Madeleine) et sept (interlocuteurs de Léonard) fois plus de futur périphrastique que de futur simple. Il semblerait donc que ces deux enfants amplifient très fortement à cet âge la tendance des adultes. Il s'agit là d'une confirmation toute quantitative de l'importance du langage entendu et du langage adressé dans l'appropriation des formes chez l'enfant.

Mais qu'en est-il des usages de ces différentes formes? En effet, si l'emploi du futur simple est moindre que celui du futur périphrastique à la fois chez les adultes et chez les enfants, il existe. Cela semble indiquer que dans la nouvelle génération, au moins chez les parisiens de classe moyenne, il perdure. Nous avons donc voulu nous intéresser aux spécificités fonctionnelles de ces formes, à leurs éventuelles différences et à leurs usages en contexte à la fois chez les adultes et les enfants afin de mieux caractériser ces deux types de futur dans le langage enfantin.

Nous avons procédé au codage de tous les énoncés dans lesquels les mères ou les enfants emploient du futur périphrastique et futur simple. Ce codage vise à faire une analyse des oppositions entre les usages du futur périphrastique et du futur simple et à vérifier si ces oppositions existent, si elles sont utilisées de manière systématique et si les usages de l'adulte et de l'enfant se recouvrent.

Nous avons examiné chez l'adulte et chez l'enfant le rapport entre les formes du futur et le contexte extra-linguistique de leur production. Nous avons codé 
DISC quand il y avait une rupture entre l'actuel - qui est défini comme ce qui se passe dans l'espace-temps dans lequel se trouve l'enfant - et l'action exprimée dans l'énoncé au futur. Cela correspondrait à ce que ce que Damourette \& Pichon (1968, § 1709) appellent "le sens toncal" :"Le placement du fait verbal dans une autre sphère d'action, une autre actualité, que celle où se trouve le locuteur au moment de la parole." Quand par contre, les productions sont ancrées dans la situation, dans l'actuel, nous avons codé CONT quand il y avait continuité avec les activités en cours et CHG quand il y avait changement. Nous avons par ailleurs codé en notant +LOC la présence de marqueurs de localisations temporelles qui peuvent être adverbiales (demain, tout à l'heure, après) ou sous forme de propositions subordonnées (quand tu seras plus grand), ») +MOD la présence de marqueurs de modalité (par exemple pouvoir, ou « il faut »), et +ARG la présence de marqueurs d'argumentation (sinon, parce que, comme ça...). Nous avons également codé REP quand les énoncés étaient des reprises d'un énoncé précédent de l'adulte, et IND quand il ne nous était pas possible de coder. Nous avons également codé la personne employée pour chaque énoncé au futur. 


\section{VALEURS}

Notre codage nous a permis d’obtenir les résultats suivants.

\begin{tabular}{|c|c|l|c|c|c|c|c|c|}
\hline Leonard & & Total & CONT & CHG & DISC & REP & IND \\
\cline { 2 - 9 } & \multirow{2}{*}{$\begin{array}{c}\text { Enfa } \\
\text { nt }\end{array}$} & Futur peri & 118 & 42 & 17 & 36 & 16 & 7 \\
\cline { 2 - 9 } & $\begin{array}{c}\text { Adul } \\
\text { tes }\end{array}$ & Futur simple & 8 & 0 & 0 & 8 & 0 & 0 \\
\cline { 3 - 10 } & Futur péri & 300 & 134 & 97 & 60 & 9 & 0 \\
\hline \multirow{2}{*}{ Madeleine simple } & 41 & 4 & 5 & 32 & 0 & 0 \\
\hline & $\begin{array}{c}\text { Enfa } \\
\text { nt }\end{array}$ & Futur péri & 250 & 169 & 43 & 31 & 5 & 2 \\
\cline { 2 - 10 } & $\begin{array}{c}\text { Adul } \\
\text { tes }\end{array}$ & Futur Simple & 19 & 2 & 1 & 13 & 2 & 1 \\
\cline { 3 - 10 } & Futur péri & 261 & 205 & 33 & 20 & 0 & 3 \\
\hline
\end{tabular}

Tableau 1 : Résultat du codage en valeurs absolues

\begin{tabular}{|c|c|l|c|c|c|}
\hline \multirow{3}{*}{ Leonard } & & & CONT & CHG & DISC \\
\hline \multirow{2}{*}{ Enfant } & Futur péri & $44 \%$ & $18 \%$ & $38 \%$ \\
\cline { 3 - 6 } & & Futur simple & $0 \%$ & $0 \%$ & $100 \%$ \\
\cline { 3 - 6 } & \multirow{2}{*}{ Adultes } & Futur péri & $46 \%$ & $33 \%$ & $21 \%$ \\
\cline { 3 - 6 } & & Futur simple & $10 \%$ & $12 \%$ & $78 \%$ \\
\hline \multirow{2}{*}{ Madeleine } & \multirow{2}{*}{ Enfant } & Futur péri & $70 \%$ & $18 \%$ & $13 \%$ \\
\cline { 3 - 6 } & & Futur simple & $13 \%$ & $6 \%$ & $81 \%$ \\
\cline { 2 - 6 } & \multirow{2}{*}{ Adultes } & Futur péri & $79 \%$ & $13 \%$ & $8 \%$ \\
\cline { 3 - 6 } & & Futur simple & $0 \%$ & $9 \%$ & $91 \%$ \\
\hline
\end{tabular}

Tableau 2 : Résultat du codage en pourcentage pour les formes CONT, CHG et DISC

Dans l'analyse de ces résultats, on est d'abord frappé par les différences individuelles et par la similitude entre les profils de l'adulte et de l'enfant dans la dyade. Ainsi, la mère de Léonard emploie beaucoup moins le futur simple que la mère de Madeleine, et les enfants semblent répliquer cette différence entre les mères. Par ailleurs, les répartitions des différents emplois du futur périphrastique sont similaires chez les mères et leur enfant.

Très clairement, ce codage montre également que le futur simple a un usage bien spécifique et circonscrit chez les adultes et chez les enfants alors que le futur périphrastique semble couvrir tout un ensemble d'usages. En effet, la quasi-totalité des futurs simples du corpus sont codés par DISC, c'est-à-dire que les énoncés ont été interprétés comme étant en discontinuité ou rupture temporelle ou énonciative avec la situation d'énonciation. Cet emploi du futur simple est suffisamment spécifique et caractéristique pour être repris par les enfants à l'identique. Ainsi, même lorsqu'une forme est rare, sa spécificité fait sa " force » énonciative, et peut être suffisante pour que son usage soit repris par l'enfant. A l'inverse, pour ce qui concerne les emplois du futur périphrastique, le tableau est très différent. Les formes et structures les plus fréquemment utilisées, 
de façon plus généralisée, moins circonscrite, et avec des usages plus ambigus et variés, posent des problèmes d'interprétation.

Après cette présentation des résultats, nous allons essayer de les expliquer en nous penchant de manière approfondie sur des exemples d'emplois du futur simple et du futur périphrastique chez les deux enfants en comparant leurs énoncés et ceux de leurs interlocuteurs-adultes.

\section{LE FUTUR SIMPLE}

Dans nos données, Léonard commence à employer le futur à 2;11 dans des contextes où la forme est très souvent provoquée par des questions et sollicitations adultes comportant des futurs simples. Léonard semble cependant clairement utiliser le futur dans des situations de rupture temporelle.

Exemple 1 : Léonard 2;11

*MOT: et Léonard qu'est-ce-que tu feras quand tu seras grand ?

${ }^{*} \mathrm{CHI}$ : on fera la cuisine.

Cet exemple est intéressant, car le futur est induit par la question de la mère qui emploie à la fois ce temps grammatical et une subordonnée temporelle qui projette l'enfant dans une situation décrochée de son présent, sans pourtant définir un repère temporel précis. Il s'agit davantage d'inactuel que d'une réelle localisation temporelle. Il y a donc un repérage inter-événements entre « fera la cuisine» et «seras grand». Dans sa réponse, l'enfant se situe dans ce plan aoristique, parle de « faire la cuisine », et change de personne ce qui suppose une certaine maîtrise de la forme verbale puisqu'il a recours au « on » (comme s'il associait sa mère à ce projet), ce qui suppose une réappropriation de ce mode de représentation.

On trouve également dans cette période qui va de 2;11 à 3;02 des utilisations spontanées du futur simple et même des hésitations au niveau de la morphologie verbale qui montrent bien que l'enfant est conscient de devoir produire une forme spécifique.

Exemple 2: Léonard 3;0

*OBS: qu'est-ce-que vous allez faire là-bas ?

*CHI: et puis et puis et puis moi je jouais .

*CHI: et moi je je jouais

*CHI: moi je joueRA !

On peut noter que l'observateur pose une question en employant un futur périphrastique. La réponse de l'enfant est intéressante à un double niveau. On observe d'abord une forme qui, phonétiquement, est un imparfait sur le verbe jouer, et qui s'insère dans une narration linéaire marquée par les et puis, puis dans un deuxième temps, l'enfant reformule sa forme verbale plusieurs fois 
jusqu'à ce qu'il parvienne à produire une forme de futur avec un accord qui correspond à la troisième personne au lieu de la première. Cette " erreur » montre de façon encore plus frappante qu'il avait bien une cible à atteindre : produire une forme de futur.

L’hésitation imparfait/futur est peut-être révélatrice d'un fonctionnement commun entre les deux formes au niveau du décrochage narratif des évènements décrits par rapport à l'actualité de l'enfant, ce qui correspond bien à la valeur codée DISC.

En comparaison, Madeleine démontre un usage beaucoup plus précoce et plus autonome du futur simple. Elle commence par utiliser la forme à 2;03 dans un contexte narratif (une histoire qu'elle connaît par cœur) ce que l'on peut rapprocher de nos remarques sur les liens entre les emplois du futur et du passé dans la narration. Très rapidement cependant, on trouve des occurrences spontanées.

Ses premières occurrences peuvent être incomplètes grammaticalement (en particulier pronom personnel manquant), mais sont indubitablement des futurs.

Exemple 3 : Madeleine 2;04

@sit: Madeleine est dans une situation de jeu symbolique avec ses jouets et raconte...

*CHI: alors \# demanderai \# de faire un service.

Madeleine utilise le futur soit pour parler d'évènements repérés, souvent explicitement, dans un avenir assez lointain et sans lien avec la situation d'énonciation, soit dans le jeu symbolique qui lui permet de se projeter et de raconter ce qui selon elle devrait advenir.

Exemple 4 : Madeleine 2;03. Elle vient d'avoir un petit frère.

*OBS: bébé Côme tu l'as vu déjà ?

*CHI: hum .

*OBS: il est mignon il est beau ?

*CHI: moi je le verra c'est l'après-midi.

*OBS: cet après-midi ?

*CHI: moi je le verra c'est ${ }^{5}$ l'après [//] c'est l'après-midi.

En dépit des erreurs de réalisation du futur (erreur flexionnelle) et de l'adjectif démonstratif sans doute imparfaitement prononcé (/se/ au lieu de /set/), Madeleine produit une marque explicite de positionnement temporel «c'est l'après-midi ». Dans les exemples qui suivent, l'ancrage futur est réalisé par les subordonnées temporelles, mais qui définissent davantage des contextes de validation qu'elles ne sont de réelles indications temporelles précises.

Exemple 5 : Madeleine 2;08 explique ce qui se passera quand elle sera dans la classe des verts à la maternelle.

${ }^{5}$ La transcription orthographique suggère la production de «c'est » mais il peut aussi s'agir d'un écart dans la réalisation du démonstratif « cet ». 
*CHI: mais tu sais quand je s(e)rai yy vert tu m(e) prendras <en pho(to)> [///] en caméra maman et doudou et Côme et toi et moi et Lucas et mon doudou euh euh tu m(e) prendras en caméra .

Exemple 6 : Madeleine 2;09

*MOT: et est-ce-que tu as raconté nos vacances de Noël à Martine .

*MOT: tu lui as raconté ou pas?

*CHI: non j(e) lui raconte pas parce que $<$ c'est pas $>$ [///] c'est un secret .

*CHI: <on lui $>$ [/] on lui dira quand ce s(e)ra plus un secret.

Les emplois du futur simple offrent peu de divergence entre les deux enfants en dépit des différences de précocité dans leur développement langagier. En effet, tous deux restreignent les emplois du futur simple à des procès clairement en rupture temporelle ou parfois modale avec la situation d'énonciation. Le futur simple est d'ailleurs fréquemment employé sur des verbes modaux suivis de l'infinitif et pas n'importe lesquels, puisqu'il s'agit principalement des verbes falloir et pouvoir utilisés sans sujet:

Exemple 7 : Madeleine 2;07 (à propos d'un ours)

*MOT : tu vois, les grandes oreilles, c'est un drôle d'ours non ?

*CHI : il a de grand[//] grand [/] grand du coup faut [///] faudra lui acheter du [//] des lunettes.

La récurrence de ces verbes particuliers permet à l'enfant de marquer une nécessité ou une possibilité extérieure à l'enfant, et détachée de son intention ou de sa volonté. Ce décrochage modal est confirmé dans cet exemple par l'expression « du coup » qui fait un lien direct entre les grandes oreilles et la nécessité des lunettes.

Lorsqu'on observe les emplois des adultes, on constate que tous les futurs simples sont non seulement en rupture (DISC), mais presque systématiquement accompagnés d'un marquage temporel, modal ou argumentatif (en conformité avec les analyses de Jeanjean 1988).

\section{LE FUTUR PERIPHRASTIQUE}

Les emplois du futur périphrastique étant plus variés et complexes, nous présentons ci-dessous des graphiques illustrant les différentes valeurs qu'il prend dans les énoncés des deux enfants. 


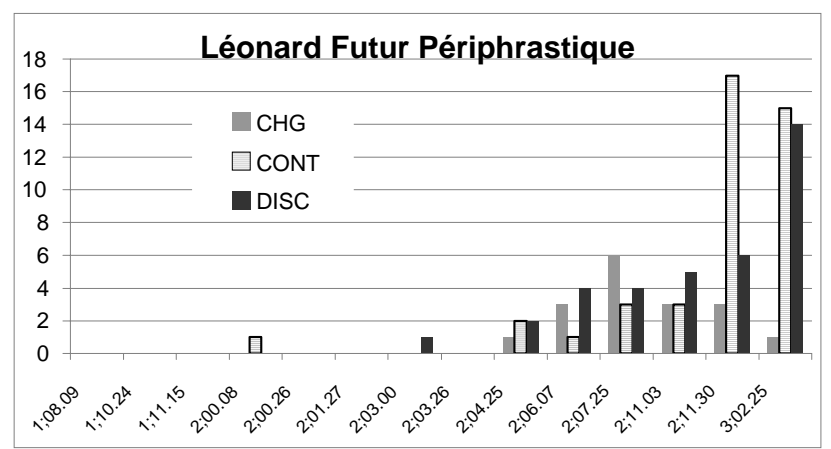

Graphique 3 : les différentes valeurs du futur périphrastique chez Léonard

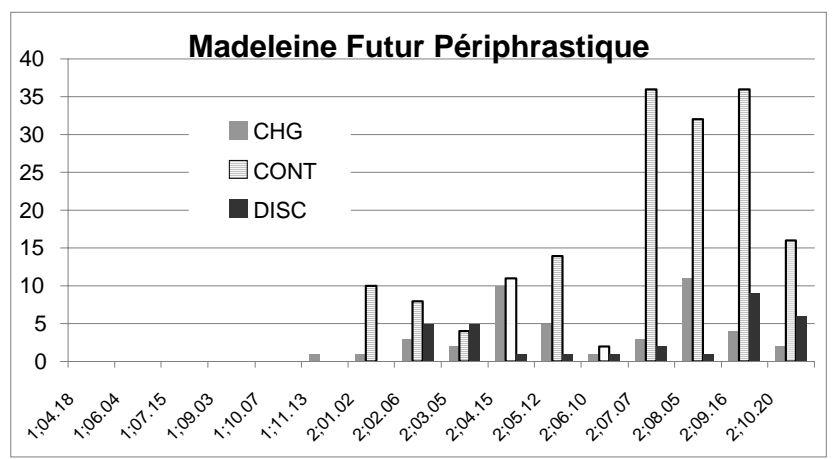

Graphique 4 : les différentes valeurs du futur périphrastique chez Madeleine

On constate que l'utilisation variée du futur périphrastique apparaît dès son émergence dans le corpus. On remarque, tout comme pour les emplois du futur simple, que Léonard produit une partie de ses formes en répétition ou en expansion de l'adulte, alors que Madeleine manifeste immédiatement son autonomie langagière en produisant des formes non induites.

Madeleine a une utilisation préférentielle du futur périphrastique qui marque le plus souvent chez elle un procès en continuité (CONT) avec la situation en cours. Par ailleurs, chez les deux enfants, les formes au futur périphrastique sont majoritairement à la première personne (46 \% chez Léonard et 55\% chez Madeleine) alors que l'on ne trouvait pas cette tendance au futur simple (25\% chez Léonard et 31\% chez Madeleine). Ces deux observations indiquent une mise en relation privilégiée des évènements futurs avec le point de vue énonciatif temporel et subjectif de l'enfant lorsque celui-ci produit un futur périphrastique. Ce rattachement aspecto-modal au point de vue de l'enfant est confirmé par les nombreuses alternances entre «je veux faire » et «je vais 
faire », qui soulignent la proximité entre le désir et la prise en charge de son accomplissement. A 2;06 par exemple, Léonard, en parlant d'un tableau, associe les deux formes : « je veux, je vais essuyer ». Notons que « Je vais essuyer » est plus télique que « je veux essuyer ». Il n'y a plus seulement l'expression d'un désir, mais prédication d'une décision d'action, souvent polémique par rapport à la position de l'adulte.

Voici une illustration des trois valeurs codées dans les énoncés contenant des futurs périphrastiques chez les enfants.

CONT - Exemple 8 : Léonard 2;07 est en train de lire un livre avec l'observatrice.

*OBS: et il a mal tu crois?

*CHI: ouais il a mal. lui i va mettre un pansement à lui.

CHG - Exemple 9 : Madeleine 2;09

*OBS: bonjour .

*CHI: attend .

*MOT: tu sais Madeleine.

*CHI: $\mathrm{j}(\mathrm{e})$ vais prendre un habit pour [/] pour [/] pour Charlotte.

DISC - Exemple 10 : Madeleine 2;02

*CHI: l' est là .

*MOT: ce livre $\mathrm{xxx}$ fait encore .

*CHI: tu peux lire ?

*CHI: (a)près [//] après on va finir \# le jeu .

Il est intéressant de constater que si la valeur DISC n’est pas réservée au futur simple, quand on la trouve pour le futur périphrastique, elle est accompagnée d'un marquage temporel, modal ou argumentatif. Le fait que l'on puisse avoir la valeur DISC avec les deux formes de futur, nous amène à faire une distinction plus fine entre deux façons de représenter une discontinuité entre l'action exprimée et l'actualité. D’une part, les énoncés en discontinuité au futur périphrastique sont en général repérés dans un futur moins distant que les énoncés au futur simple (ce qui semble recouper la distinction futur lointain/ futur proche, mais n'est pas suffisant). D'autre part on observe, dans l'exemple 10, que le fait de finir le jeu est reporté à plus tard, mais qu'un lien de successivité temporelle est posé (après). Par ailleurs, ce futur périphrastique nous paraît accentuer la volonté et l'intention de l'enfant d'évoquer, de son point de vue dans l'activité en cours (la lecture d'un livre), une activité désirée mais autre. Malgré la distanciation entre l'évocation verbale et la réalisation de l'action "finir le jeu », l'enfant rattache cette activité à son point de vue temporel et modal présent (son désir, sa volonté). Il s'agit donc d'un cas de DISC dans lequel l'enfant produit une forme non aoristique qui s'apparente davantage à une forme aspecto-modale. On observe d'ailleurs le même phénomène dans «l'input» adulte. La mère de Madeleine commente les mouvements de sa fille en disant « là, on se dit qu'un jour elle va se cogner ». Il y a à la fois un décrochage par rapport à l'actualité (un jour) et une mise en relation avec ce que Madeleine vient de faire « là » que l'on peut opposer à un 
énoncé au futur simple «un jour elle se cognera » qui définirait plutôt une évidence quasi scientifique non liée au point de vue subjectif de la mère.

Une fois qu'elles sont maîtrisées par les enfants, les deux formes de futur se font-elles donc concurrence ? Il pourrait sembler, comme le montre Laurendeau (2000) pour le français canadien, que les marqueurs coexistent mais " construisent des formes sémantico-énonciatives distinctes ». En tant que linguistes cherchant à repérer des invariants des différentes formes utilisées, on peut donc associer l'usage du futur périphrastique chez l'enfant à une prise en charge plus grande. Il s'agirait d'un moyen d'actualiser un procès à venir et de faire « oublier » la valeur épistémique, l'incertitude quant à la validation de la relation prédicative.

Exemple 11: Madeleine 2;10

${ }^{*} \mathrm{CHI}$ : On va boire de l'eau comme ça

\%act : Elle mime.

Madeleine semble actualiser le procès avec le futur périphrastique ce qu'elle double en mimant l'action.

Exemple 12 : Léonard 2;06

*MOT: A. fait un film pour voir comment Léonard parle.

*MIN: ah !

*CHI: après i va prêter.

Léonard veut emprunter la caméra à l’observatrice et son énoncé manifeste explicitement son désir d'actualiser le moment à venir où celle-ci la lui prêtera.

Le fait que ces formes véhiculent des valeurs différentes ne signifie cependant pas que l'enfant ferait un choix conscient des formes par rapport à ces valeurs, mais qu'il y a une intégration d'un système de langue qu'il trouve dans l'input langagier, et réappropriation progressive. La fréquence des emplois chez les adultes peut aussi expliquer la distribution des usages enfantins. On peut considérer que le futur périphrastique, quinze fois plus utilisé dans nos données que le futur simple, serait la valeur non marquée, utilisée un peu « par défaut ».

L'analyse des formes verbales de futur dans deux suivis longitudinaux d'interaction adulte-enfant français nous montre tout d'abord que le futur simple reste un mode pertinent pour exprimer l'avenir en français contemporain en dépit de l'emploi quatre à cinq fois plus important chez les adultes du futur périphrastique. La spécificité même de l'usage du futur simple, que les enfants comme les adultes emploient dans un contexte de discontinuité avec la situation d'énonciation, pourrait être le garant de sa survie. En effet, on constate en général en diachronie un phénomène de persistance des formes, lexicales ou grammaticales, dont l'usage est très spécifique, comme c’est le cas, par exemple, pour les verbes irréguliers. Par ailleurs, les variations d’usage du futur des 
enfants correspondent à celles des adultes avec lesquels ils interagissent. Ceci est particulièrement frappant pour le futur périphrastique dont les emplois sont variés. On constate également que cette variété incite à utiliser d'autres marqueurs lexicaux ou syntaxiques pour clarifier les valeurs, qu'elles soient d'ordre temporel, argumentatif ou modal.

Notre étude montre également qu'il y a de grandes variations entre langues comme on peut le constater dans la comparaison des formes employées par les enfants en français et en anglais (d'après le travail de Gee 1985) et il semble y avoir des rapprochements et des différences entre «will» et le futur périphrastique, ainsi qu'entre "gonna » et le futur simple. Chaque langue a son propre système dans lequel les équilibres entre temps, aspect et modalité se font de manière différente.

Ainsi, l'analyse des formes qui permettent d'exprimer le futur peut nous apporter des éclairages très intéressants, non seulement sur ces formes ellesmêmes, mais également sur la manière dont l'enfant s'approprie le langage. En effet, il s'agit d'emplois relativement peu fréquents, qui sont donc peu automatisés et qui laissent place à l'initiative de l'enfant. 


\section{BIBLIOGRAPHIE}

Applebee, A.N. (1978). The childs's concept of story: age two to seventeen. Chicago, The university of Chicago Press.

Celle, A. (1994) « La traduction de will ». Linguistique contrastive et traduction. Tome 3. Paris, Gap : Ophrys. 87-139.

Celle, A. (1997) Etude contrastive du futur français et de ses réalisations en anglais. Paris, Gap : Ophrys.

Celle, A. (2003) « La visée dans les propositions hypothétiques en anglais et en français " in Celle, A. \& S. Gresset (eds). La subordination en anglais, une approche énonciative. Toulouse : Presses Universitaires du Mirail.

Comrie, B, (1985). Tense. Cambridge Textbooks in Linguistics. Cambridge, Cambridge University Press.

Damourette, J. \& Pichon, E. (1911-1933) Des mots à la pensée, essai de grammaire de la langue française, Paris: réed. d'Artrey (1968).

Franckel, J. J. (1984). Futur «simple» et futur «proche», Le français dans le monde, $\mathrm{n}^{\circ} 182$, pp. 65-70.

François, F. (1984). Problèmes et esquisse méthodologique, in François, F., Hudelot, C., \& Sabeau-Jouannet, E., (1984), Conduites linguistiques chez le jeune enfant. Paris : P.U.F, 13-116.

Gee, J. (1985). An interpretive approach to the study of modality : what child language can tell the linguist. Sutdies in Language 9-2, 197-229.

Grégoire, A. (1947). L'apprentissage du langage, la troisième année et les années suivantes, Paris, Alcan.

Guillaume, P. (1924). Les débuts de la phrase dans le langage de l'enfant, Journal de Psychologie Normale et Pathologique, 1-25.

Harner, L. (1981). Children's talk about the time and aspect of actions. Child development, 52, 498-506.

Herriot, P. (1969). The comprehension of active and passive sentences as a function of pragmatic expectations. Journal of verbal learning and Verbal behavior. 8 (2), 166-169.

JeanJean, C. (1988). Le futur simple et le futur périphrastique en français parlé, Blanche-Benveniste, C.; Chervel, A.; Gross, M. dir. Grammaire et histoire de la grammaire: Hommage à la mémoire de Jean Stéfanini, Publications de l'Université de Provence, pp. 235-257.

Laurendeau, P. (2000). L'alternance futur simple, futur périphrastique : une hypothèse modale. Verbum, tome 22, fascicule 3, pp 277-292.

Lyons, J. (1978, traduction 1980). Sémantique Linguistique. Traduction J. Durand et D. Boulonnais. Larrousse Université.

MacWhinney, B. (2000). The CHILDES Project: Tools for analyzing talk, 3rd Edition. Vol. 2: The Database, Mahwah, NJ, Lawrence Erlbaum Associates.

Malrieu, Ph. (1953), Les origines de la conscience du temps, les attitudes temporelles chez l'enfant, Parts PUF.

Palmer, F.R. (1979). Modality and the English Modals. London and New-York : Longman.

Piaget, J. (1916). Le développement de la notion de temps chez l'enfant. Paris, PUF, $2^{\text {eme }}$ édition, 1973.

Piaget, J. (1937). La construction du réel chez l'enfant, Neuchâtel, Delachaux et Niestlé. 
Sabeau-Jouannet, E. (1977). L'expression de l'organisation spatiale et temporelle, son évolution chez l'enfant de 2 à 5 ans, in F. François (Ed.) $L a$ syntaxe de l'enfant avant cinq ans, Larousse.

Wallon, H. (1945). Les origines de la pensée chez l'enfant. Paris, PUF, $3^{\circ}$ édition, 1963. 